\title{
Measuring Inflation Expectations Uncertainty Using High-Frequency Data
}

\author{
Joshua C.C. Chan* \\ Economics Discipline Group, \\ University of Technology Sydney,
}

\author{
Yong Song \\ Department of Economics, \\ University of Melbourne
}

September 2017

\begin{abstract}
Inflation expectations play a key role in determining future economic outcomes. The associated uncertainty provides a direct gauge of how well-anchored the inflation expectations are. We construct a model-based measure of inflation expectations uncertainty by augmenting a standard unobserved components model of inflation with information from noisy and possibly biased measures of inflation expectations obtained from financial markets. This new model-based measure of inflation expectations uncertainty is more accurately estimated and can provide valuable information for policymakers. Using US data, we find significant changes in inflation expectations uncertainty during the Great Recession.
\end{abstract}

Keywords: trend inflation, inflation expectations, stochastic volatility

JEL Classification: C11, C32, E31

* Joshua Chan would like to acknowledge financial support by the Australian Research Council via a Discovery Project (DP170101283). 


\section{Introduction}

Inflation expectations play a key role in price and wage setting behavior, and therefore have a substantial influence on future economic outcomes. Policymakers and central bankers in particular pay close attention to measures of long-run inflation expectationsthese expectations reveal information about the credibility of monetary policy and are an important input in the formulation of macroeconomic policy in general. Given their importance, there is now a large and growing literature on combining econometric models of trend inflation with inflation expectations from surveys of professionals or consumers to obtain better estimates of inflation expectations or better inflation forecasts. Prominent examples include Kozicki and Tinsley (2012), Wright (2013), Nason and Smith (2013) and Mertens (2016).

Building on this line of research, we investigate the information content of market-based measures of inflation expectations for refining estimates of inflation expectations volatility or uncertainty. ${ }^{1}$ Such a measure is useful for several reasons. Monetary policy tools work differently if inflation expectations are firmly anchored than if they are not. In particular, monetary policy is thought to be most effective when inflation expectations are stable. Hence, a measure of inflation expectations uncertainty provides a direct gauge of how wellanchored the inflation expectations are. This measure can be used to assess, for example, the effectiveness of forward guidance. A more refined measure of inflation expectations uncertainty can also be used to develop a better second moment policy such as a financial stabilization package to reduce systemic risk.

To construct such a measure of inflation expectations uncertainty, we develop a new bivariate unobserved components model. We take a model-based approach and combine direct measures of inflation expectations uncertainty and information in model-based estimates. In essence, we aim to view these direct measures of inflation expectations uncertainty through the lens of an econometric model. Our point of departure is the univariate unobserved components model with stochastic volatility (UCSV) of Stock and Watson (2007) that is widely used to model inflation (see, e.g., Chan, Koop, and Potter, 2013; Clark and Doh, 2014). Under some assumptions, trend inflation from this model should correspond to long-run inflation expectations. Hence, the time-varying volatility of trend inflation can be interpreted as long-run inflation expectations uncertainty.

\footnotetext{
${ }^{1}$ Here the uncertainty is about long-run inflation expectations, which is different from uncertainty about long-run inflation.
} 
We augment this model-based measure of uncertainty with information from marketbased inflation expectations. Specifically, we obtain breakeven inflation computed from long-horizon real and nominal bonds, which is available daily. We then compute the associated realized volatility (see, e.g., Andersen et al., 2003), say, within a month. The constructed realized volatility gives a quantitative measure of the variation of inflation expectations, but it may also reflect other idiosyncratic factors such as volatility of risk premiums. As such, it may not be appropriate to directly equate the realized volatility with the volatility of inflation expectations. However, we can still incorporate this additional information into the UCSV model by adding a new measurement equation that relates the realized volatility to the latent time-varying volatility of trend inflation. Using this bivariate model, we can extract useful information in the realized volatility to refine estimates of trend inflation volatility.

Using US data, we find that the constructed measure of realized volatility helps improve the estimation precision of inflation expectations uncertainty compared to the benchmark UCSV model. We find significant changes in inflation expectations uncertainty during the Great Recession, in contrast to the largely flat estimates from the UCSV model. By incorporating the realized volatility, the new model is able to pick up drastic changes in inflation expectations uncertainty. Using the marginal likelihood as a model comparison criterion, we show that this new model compares favorably to the benchmark. In addition, in a pseudo out-of-sample forecasting exercise, the new model that incorporates the realized volatility measure provides better density forecasts compared to the benchmark.

Our paper is also related to the literature on measuring uncertainty and studying its impact on the economy. Since the seminal paper by Bloom (2009), many studies have contributed to this literature, including Bloom (2013), Caggiano, Castelnuovo, and Groshenny (2014), Jurado, Ludvigson, and Ng (2015) and Mumtaz and Theodoridis (2015). In particular, Berument, Yalcin, and Yildirim (2009) and Chan (2017) study the impact of inflation volatility on inflation. Our paper provides a new measure of inflation expectations volatility, and it would be interesting in future work to study its impact on other macroeconomic variables.

The rest of this paper is organized as follows. Section 2 introduces the model and discusses the interpretation of trend inflation volatility. Section 3 defines the breakeven inflation and explains how the realized volatility measure is constructed. The data and estimation are outlined in Section 4, and Section 5 presents the estimation and forecasting results using US inflation data. For robustness checks, Section 6 presents additional results using 
variations of the proposed model and different data sources. Finally, Section 7 concludes and discusses some future research direction.

\section{Modeling Trend Inflation Uncertainty}

The trend-cycle decomposition of inflation, $\pi_{t}$, is motivated by the idea that it can be usefully viewed as the sum of two separate components: a nonstationary component that represents the trend inflation, $\pi_{t}^{*}$, and a transitory deviation from the trend, or the inflation gap, $u_{t}^{\pi}$ :

$$
\pi_{t}=\pi_{t}^{*}+u_{t}^{\pi}
$$

To identify the two components, one typically makes assumptions that imply

$$
\lim _{j \rightarrow \infty} \mathbb{E}_{t} \pi_{t+j}=\lim _{j \rightarrow \infty} \mathbb{E}_{t} \pi_{t+j}^{*}=\pi_{t}^{*}
$$

and

$$
\lim _{j \rightarrow \infty} \mathbb{E}_{t} u_{t+j}^{\pi}=0,
$$

where $\mathbb{E}_{t}$ is the conditional expectation given the information at time $t$. For example, if one assumes that $\pi_{t}^{*}$ follows a random walk and $u_{t}^{\pi}$ follows a stationary $\operatorname{AR}(1)$ process with 0 mean, then both conditions are satisfied. The decomposition in (1) together with the conditions in (2) and (3) maybe seen as a generalization of the Beveridge-Nelson decomposition (Beveridge and Nelson, 1981). In our empirical work the inflation $\pi_{t}$ is in monthly frequency.

Under the conditions in (2) and (3), we may view the trend inflation $\pi_{t}^{*}$ as some longhorizon inflation expectation. Specifically, given the information at time $t$, the expected future inflation for period $t+j$ for some large $j$ should provide an estimate of $\pi_{t}^{*}$. A few recent papers have exploited this relationship and used survey long-horizon inflation expectations made at time $t$ to produce estimates of current trend inflation, with Kozicki and Tinsley (2012) and Faust and Wright (2013) being prominent examples.

Our modeling approach is similar to the one in Chan, Clark, and Koop (2017), where they extend the unobserved components model with stochastic volatility (UCSV) of Stock and Watson (2007) by incorporating survey-based long-run inflation expectations. More specifically, an additional measurement equation is added to include long-run inflation expectations, $x_{t}$, obtained from the Federal Reserve Board of Governor's FRB/US econo- 
metric model and Blue Chip Consensus. They find that that long-run inflation expectations can substantially refine estimates of trend inflation over popular alternatives. But it is inappropriate to equate trend inflation with the long-run inflation expectations.

We exploit a different source of information for a different purpose. Specifically, we investigate if the realized volatility of market-based long-horizon inflation expectations at time $t$-which we denote as $z_{t}$-provides useful information for the trend inflation uncertainty. Heuristically, because we observe the market-based measure of inflation expectations at daily frequency, we could obtain an estimate of the variance of inflation expectations at lower frequency, say, monthly, as in Andersen, Bollerslev, Diebold, and Labys (2003) and Barndorff-Nielsen and Shephard (2002a), and use it as a measure of inflation expectations uncertainty.

Barndorff-Nielsen and Shephard (2002b) apply the realized variance method to a very flexible stochastic volatility framework. In this framework, a time series $y(t)$ satisfies the stochastic differential equation

$$
d y(t)=\mu(t) d t+\sigma(t) d W(t)
$$

where $\mu(t)$ is the drift, $\sigma(t)$ is the spot volatility and $W$ is the Brownian motion. Under weak assumptions, the variance of $y(t)$ can be consistently estimated by the realized variance, which is defined as

$$
\sum_{i=1}^{q}\left(y\left(t_{i}^{q}\right)-y\left(t_{i-1}^{q}\right)\right)^{2}
$$

for any sequence of partitions $t_{0}^{q}=0<t_{1}^{q}<\ldots<t_{q}^{q}=t$ with $\sup \left(t_{i}^{q}-t_{i-1}^{q}\right) \rightarrow 0$ as $q \rightarrow \infty$. This powerful result does not require any knowledge on the process of the instantaneous volatility $\sigma(t)$ nor the drift term in the stochastic differential equation. See Barndorff-Nielsen and Shephard (2002b) for more details.

However, since the theory of realized volatility relies on infill asymptotics - where more data are collected by sampling more intensely in a fixed domain - it may not be reasonable to assume that the realized volatility measure from the daily observations would be consistent for the integrated variation at monthly frequency. But one may still view this measure as a potentially useful source of information inspired by the realized volatility theory. We will delineate data construction in more details in the next section.

We take the UCSV model of Stock and Watson (2007) and augment it with an additional measurement equation of the realized volatility $z_{t}$. More specifically, consider the 
following model:

$$
\begin{aligned}
\pi_{t} & =\pi_{t}^{*}+u_{t}^{\pi}, & u_{t}^{\pi} & \sim \mathcal{N}\left(0, \mathrm{e}^{h_{t}}\right), \\
\pi_{t}^{*} & =\pi_{t-1}^{*}+u_{t}^{\pi^{*}}, & u_{t}^{\pi^{*}} & \sim \mathcal{N}\left(0, \mathrm{e}^{g_{t}}\right), \\
h_{t} & =h_{t-1}+u_{t}^{h}, & u_{t}^{h} & \sim \mathcal{N}\left(0, \sigma_{h}^{2}\right), \\
g_{t} & =g_{t-1}+u_{t}^{g}, & u_{t}^{g} & \sim \mathcal{N}\left(0, \sigma_{g}^{2}\right), \\
\log z_{t} & =a_{0}+a_{1} g_{t}+u_{t}^{z}, & u_{t}^{z} & \sim \mathcal{N}\left(0, \sigma_{z}^{2}\right),
\end{aligned}
$$

where $h_{t}$ and $g_{t}$ are respectively the log volatility of the transitory and trend components.

The UCSV model of Stock and Watson (2007) is defined by (4)-(7). The new equation is (8), which relates the log realized volatility measure to $g_{t}$, the log volatility of the trend inflation. Since $z_{t}$ is likely to be a noisy and potentially biased measure of trend inflation volatility, the measurement equation (8) allows us to estimate the relationship instead of imposing equality. For example, by allowing the parameters $a_{0}$ and $a_{1}$ to be estimated, we can investigate whether equating the log realized volatility measure with the log volatility of trend inflation is a sensible thing to do. In particular, if $\log z_{t}$ is an unbiased measure, we would expect that $a_{0}=0$ and $a_{1}=1$.

In principle one could entertain an additional measurement equation that relates a marketbased long-term inflation expectation measure (e.g., the monthly average of the daily breakeven inflation) to the trend inflation $\pi_{t}^{*}$. In preliminary work we find that the performance of the model deteriorates when this additional source of information is added; there are noticeable discrepancies between the market-based measure and the modelbased trend inflation. This likely reflects the fact that breakeven inflation includes not only inflation expectations but also liquidity and inflation risk premiums. While there are a few recent papers, such as Christensen, Lopez, and Rudebusch (2010) and Grishchenko and Huang (2012), that aim to decompose the breakeven inflation into a purely inflation expectations measure and risk premiums, these approaches often involve additional modeling and assumptions about how risk is priced. We therefore do not use the level of the market-based long-term inflation expectations.

This discussion raises the question of the quality of the realized volatility constructed, given that the breakeven inflation includes liquidity and inflation risk premiums. In next section we argue that as long as the risk premiums do not change drastically within a month, the constructed realized volatility should be a reasonable estimate of the underlying volatility. Further, in Section 5 we show empirically that the constructed realized 
volatility can refine model estimates and improve inflation forecasts. Finally, as a robustness check, we construct an alternative realized volatility measure that adjusts for liquidity premium in Section 6.2, and the corresponding estimates remain essentially the same.

\section{Realized Volatility of Inflation Expectations}

In this section we discuss how our measure of realized volatility of market-based longhorizon inflation expectations $z_{t}$ is constructed. These calculations are based on the so-called breakeven inflation or inflation compensation, which is often interpreted as a measure of expected inflation. More specifically, the breakeven inflation is the inflation rate at which the investor receives the same expected return from holding either nominal or inflation-protected bonds. The breakeven inflation reflects expected inflation-and empirical studies (e.g., Gürkaynak, Levin, and Swanson, 2010a) typically interpret it as such. But it also reflects compensation investors are demanding for risks associated with the uncertainty about future inflation or liquidity differential between the real and nominal bond markets. One main goal of our paper is to investigate whether the realized volatility of this market-based inflation expectations measure is consistent with its modelbased counterpart.

Let $r_{t}^{(k)}$ represent the real interest rate on a $k$-period bond and let $i_{t}^{(k)}$ denote the corresponding nominal interest rate. The breakeven inflation between periods $k_{1}$ and $k_{2}$ with $k_{2}>k_{1}$ is calculated as

$$
\mathrm{ei}^{\left(k_{1}, k_{2}\right)}=\frac{k_{2}\left(i^{\left(k_{2}\right)}-r^{\left(k_{2}\right)}\right)-k_{1}\left(i^{\left(k_{1}\right)}-r^{\left(k_{1}\right)}\right)}{k_{2}-k_{1}} .
$$

This quantity is commonly used as a measure of expected inflation between periods $k_{1}$ and $k_{2}$. For example, if $k_{1}=2$ and $k_{2}=3$, then ei ${ }^{(2,3)}$ may be interpreted as the average expected inflation between year 2 and 3 . One often uses ei ${ }^{(5,10)}$ as a measure of longhorizon inflation expectations, although other measures are also used (see, e.g., Jochmann, Koop, and Potter, 2010). In our empirical work, the real and nominal interest rates are the US real and nominal Treasury security yields taken from the Treasury InflationProtected Securities (TIPS) market. For further discussion of the TIPS market, see, e.g., Potter and Rosenberg (2007). We use these daily long-horizon inflation expectations to construct the relevant realized volatility. 
The construction of realized volatility for inflation expectations is complicated by the fact that this market-based measure of inflation expectations does not only reflect inflation expectations, but also includes risk premiums associated with future inflation uncertainty as well as the difference in liquidity between the nominal and real bond markets. Hence, using the level of breakeven inflation as a measure of inflation expectations might be problematic. However, if such risk premiums are constant within a month, they can be removed by using demeaned data.

Specifically, let $\mathrm{ei}_{t, i}^{\left(k_{1}, k_{2}\right)}$ denote the breakeven inflation on the $i$-th day in month $t$ and write

$$
\mathrm{ei}_{t, i}^{\left(k_{1}, k_{2}\right)}=\pi_{t, i}^{\left(k_{1}, k_{2}\right)}+\phi_{t}
$$

where $\pi_{t, i}^{\left(k_{1}, k_{2}\right)}$ is the expected average inflation between periods $k_{1}$ and $k_{2}$ given the information on the $i$-th day in month $t$ and $\phi_{t}$ is a risk premium term that is independent of $i$. Then, it is easy to see that $\mathrm{ei}_{t, i}^{\left(k_{1}, k_{2}\right)}-\overline{\mathrm{ei}}_{t}^{\left(k_{1}, k_{2}\right)}$ is independent of $\phi_{t}$, where $\overline{\mathrm{ei}}_{t}^{\left(k_{1}, k_{2}\right)}$ is the average of the daily observations within month $t$. Therefore, we construct the realized volatility by using the demeaned quadratic variation as

$$
z_{t}=\frac{1}{n_{t}} \sum_{i=1}^{n_{t}}\left(\mathrm{ei}_{t, i}^{\left(k_{1}, k_{2}\right)}-\overline{\mathrm{ei}}_{t}^{\left(k_{1}, k_{2}\right)}\right)^{2}
$$

where $n_{t}$ is the number of daily observations in month $t$.

In the context of high-frequency financial data (e.g., stock returns observed every 5 minutes), the quadratic variation is a simple estimator for the daily volatility that has good properties. In particular, since the quadratic variation of continuous finite-variation process is zero (see, e.g., property (ii) of Proposition 2 in Andersen et al., 2003), the mean component becomes irrelevant for the quadratic variation. However, these results are based on infill asymptotics which might not apply in our context as there are on average only 22 daily observations in each month. ${ }^{2}$

This discussion is meant to provide a motivation for the use of an imperfect, noisy indicator to enrich the UCSV model. As such, in the measurement equation (8) for $z_{t}$, we allow for the potential bias associated with this measurement problem. The condition that risk premiums remain constant within the month might still be too restrictive in

\footnotetext{
${ }^{2}$ In addition to the quadratic variation discussed above, other realized volatility construction methods such as bipower variation (Barndorff-Nielsen and Shephard, 2004), flat-top realized kernel (BarndorffNielsen et al., 2008) and non-negative realized kernel (Barndorff-Nielsen et al., 2011) are proposed to deal with jumps and microstructure noise. All these methods rely on infill asymptotics, and no simple small sample properties are available to the best of our knowledge.
} 
practice. In Section 6.2 we investigate whether the presence of liquidity premium distorts our measure of realized volatility. By using an alternative measure of realized volatility that adjusts for liquidity premium, we conclude that the variation in liquidity premium does not substantially affect the estimation results. In addition, we show in Section 5 that the constructed realized volatility helps refine the estimates and improve inflation forecasts.

\section{Data and Estimation}

In this section we describe the data source and outline the posterior sampler. The daily real and nominal interest rates used to compute our measure of realized volatility of inflation expectations are the US real and nominal Treasury security yields taken from the Treasury Inflation-Protected Securities (TIPS) market. Since bond yields from the TIPS market are only available relatively recently, we restrict our sample to January 2003 to December 2015. We use annualized US monthly CPI inflation rate as our inflation measure. In our baseline results, we use the maturities of 5 and 10 years. That is, the breakeven inflation is $\mathrm{ei}^{(5,10)}$. All data are sourced from the Federal Reserve Bank of St. Louis economic database. In Appendix B we report results based on the breakeven inflation $\mathrm{ei}^{(7,10)}$. The estimation results are broadly similar to the baseline case.

The model (4)-(8) is estimated using Markov chain Monte Carlo (MCMC) methods and the details are delineated in Appendix A. To summarize the posterior sampler, the parameters and the latent states are partitioned into five blocks:

1. $\mathbf{g}=\left(g_{1}, \ldots, g_{T}\right)^{\prime}$ is the vector of $\log$ volatility of the trend inflation;

2. $\mathbf{h}=\left(h_{1}, \ldots, h_{T}\right)^{\prime}$ is the vector of log volatility of the transitory component of inflation;

3. $\boldsymbol{\pi}^{*}=\left(\pi_{1}^{*}, \ldots, \pi_{T}^{*}\right)^{\prime}$ is the vector of trend inflation;

4. $\left(\sigma_{g}^{2}, \sigma_{h}^{2}, \sigma_{z}^{2}\right)$ is the collection of the error variances;

5. $\mathbf{a}=\left(a_{0}, a_{1}\right)^{\prime}$ is the vector of regression coefficients in the inflation expectation uncertainty equation (8).

Each block of parameters is simulated conditional on the other blocks. After discarding 
a burn-in sample, the sample of the random draws is used for inference. We refer the readers to Appendix A for technical details.

\section{Empirical Results}

Before presenting results from the model (4)-(8), we conduct a preliminary analysis to assess how well our measure of realized volatility of long-horizon inflation expectations matches some conventional estimates of inflation expectations uncertainty. To that end, we estimate the UCSV model of Stock and Watson (2007) using only inflation data, and report the estimates of the log volatility corresponding to the trend inflation. We also plot the log realized volatility constructed by using the real and nominal bond yields as discussed in Section 3. The results are reported in Figure 1.

Not surprisingly, the realized volatility is much nosier than the trend stochastic volatility estimates. There is a clear comovement between these two quantities, though they diverge in certain episodes. For example, the realized volatility sharply increases in 2008-2009 at the onset of the Great Recession. While the trend stochastic volatility estimate increases as well in that period, the rise is not as large. These observations suggest that the realized volatility measure is potentially useful in providing additional information for estimating the inflation expectations uncertainty, but it might be inappropriate to treat the realized volatility as an unbiased estimate of the trend stochastic volatility.

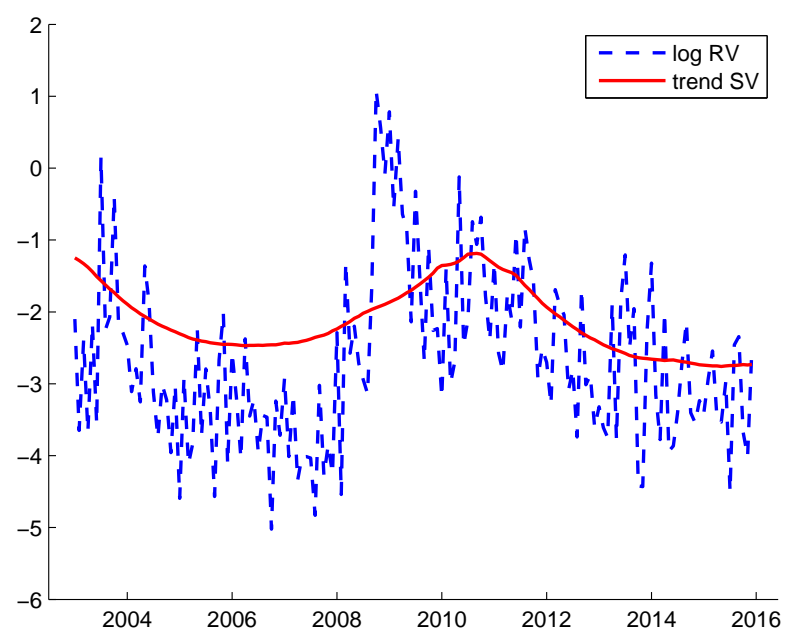

Figure 1: The log realized volatility measure and the log volatility estimates corresponding to the trend inflation under the UCSV model. 


\subsection{Inflation Expectations Uncertainty}

We first report the stochastic volatility estimates from the model (4)-(8). That is, we extend the UCSV model of Stock and Watson (2007) by incorporating the realized volatility measure $z_{t}$ as specified in (8). We refer to this model as UCSV-RV. For comparison we also present the estimates from the UCSV model. The results are depicted in Figure 2.

The most noticeable difference between the estimates from the two models occurs at the onset of the Great Recession in 2008. Under UCSV the inflation expectations uncertainty exhibits slow and gradual movements, and peaks only in 2011. This is intuitive as the UCSV model uses only inflation data; under this model the inflation expectations volatility is assumed to change slowly as specified in (7). Hence, by construction the UCSV gives smooth estimates of inflation expectations uncertainty.

In contrast, the inflation expectations uncertainty under UCSV-RV peaks in the first quarter of 2009, reflecting the sudden and drastic drop of inflation, as well as the surge of realized volatility in late 2008 and early 2009. By incorporating the realized volatility measure, the UCSV-RV model is able to pick up drastic changes in the inflation expectations uncertainty.
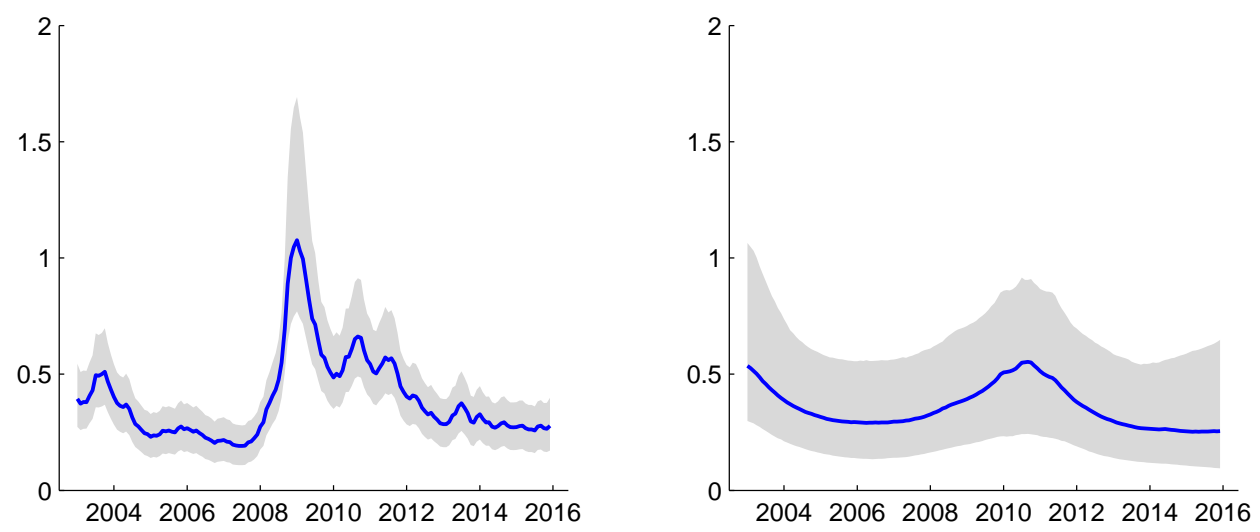

Figure 2: Stochastic volatility estimates expressed in standard deviations $\exp \left(g_{t} / 2\right)$ from UCSV-RV (left panel) and UCSV (right panel). The shaded areas represent the 16- and 84-percentiles.

In addition, Figure 2 also shows the associated $68 \%$ credible intervals of the estimates. ${ }^{3}$ Since the UCSV-RV model incorporates more information, in general the volatility of the

\footnotetext{
${ }^{3}$ Under the normal distribution, the probability of being within plus or minus one standard deviation from the mean is about 0.68 .
} 
inflation expectations is estimated more precisely compared to UCSV, as evidenced by the typically narrower credible intervals. For example, the $68 \%$ credible interval under UCSV in December 2015 is about 2.5 times wider than that of UCSV-RV.

The only exception occurs at the onset of the Great Recession, when the credible intervals of UCSV-RV become noticeably larger. This increase in uncertainty is due to the conflict between two sources of information: the realized volatility measure increases markedly at the onset of the Great Recession, whereas the state equation (7) dictates a smooth evolution of the inflation expectations volatility. Even though the former source of information dominates the posterior estimates, the model registers a higher level of parameter uncertainty.

Overall, the results show that adding the information in the realized volatility measure changes the estimates of inflation expectations uncertainty. Moreover, this additional information typically refines the inflation expectations uncertainty estimates.

Lastly, we plot the filtered estimates of the inflation expectations uncertainty in Figure 3. As expected, the filtered estimates are more volatile compared to the smoothed estimates. However, both series exhibit broadly similar trends. In particular, the filtered estimates sharply increase in early 2008 at the onset of the Great Recession. These results suggest that the model can potentially provide policymakers with timely information on inflation expectations uncertainty.

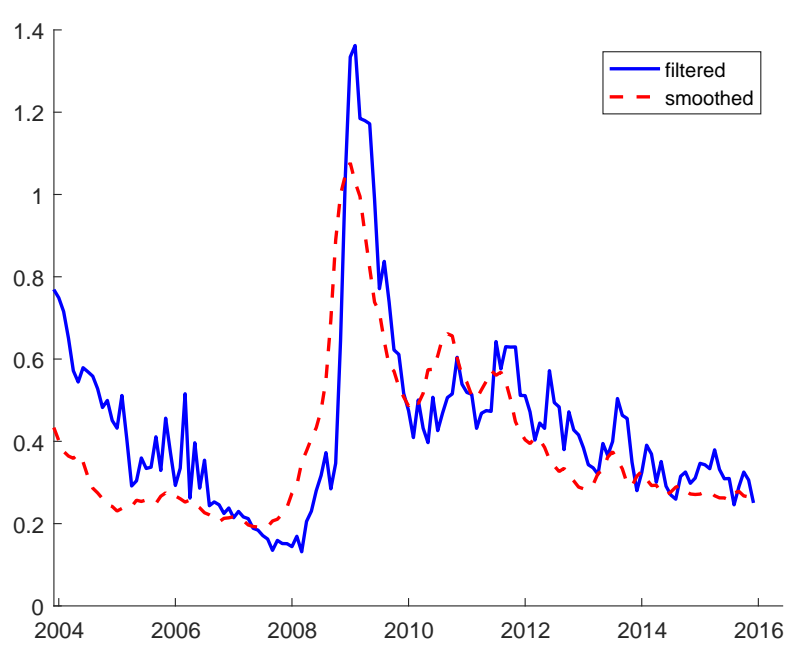

Figure 3: Stochastic volatility filtered and smoothed estimates expressed in standard deviations $\exp \left(g_{t} / 2\right)$ from UCSV-RV. 


\section{$5.2 \quad$ Inflation Expectations}

To investigate how the inclusion of realized volatility measure affects the trend inflation estimates, we report in Figure 4 the estimates from UCSV-RV. For comparison, we also present estimates from a version that also incorporates the level of breakeven inflation. Specifically, let $x_{t}$ denote the average breakeven inflation in month $t$. We then augment our model with the additional measurement equation:

$$
x_{t}=b_{0}+b_{1} \pi_{t}^{*}+u_{t}^{x}, \quad u_{t}^{x} \sim \mathcal{N}\left(0, \sigma_{x}^{2}\right) .
$$

We call this variant UCSV-RV-BE. Finally, we also report results from the UCSV model of Stock and Watson (2007).

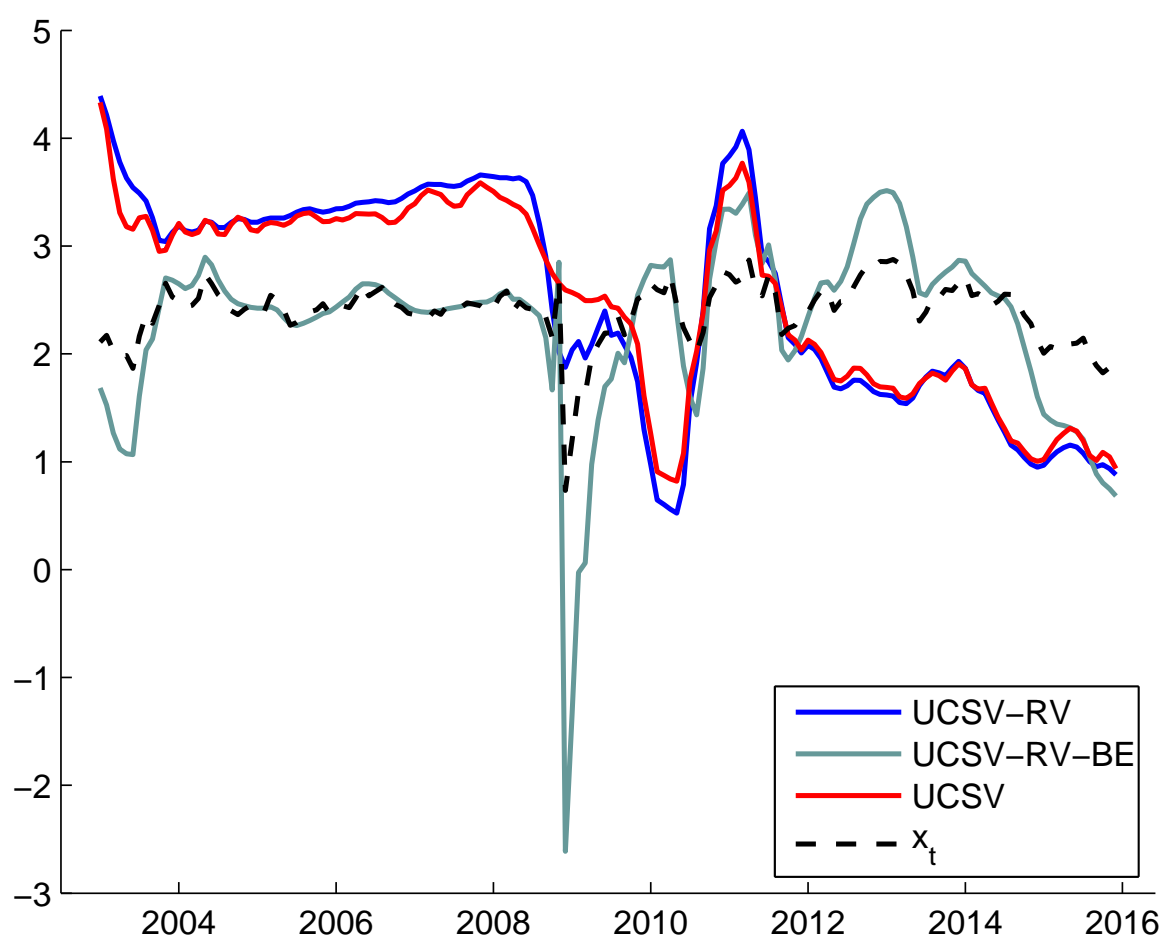

Figure 4: Trend estimates from UCSV-RV, UCSV-RV-BE and UCSV.

Comparing the estimates from UCSV-RV and UCSV, it is clear that they are remarkably similar in most of the sample. The exception is the few months in the early phase of the Great Recession. For example, in November 2008 the trend estimate under UCSVRV is about $1.9 \%$, whereas that under UCSV is $2.6 \%$. This reflects the heightened trend 
inflation or inflation expectations uncertainty under UCSV-RV, and the model gives more weight to the actual inflation data. Since the inflation rate was negative, this drags down the trend inflation estimate.

Interestingly, the estimates under UCSV-RV-BE become negative during the Great Recession, partly due to the sudden drop of the breakeven inflation. These results confirm the conclusion in Faust and Wright (2013), who warn against interpreting the breakeven inflation as a pure measure of inflation expectations. In addition, we show in Section 5.4 that UCSV-RV-BE fits the inflation data relatively poorly compared to the other two models.

\subsection{Parameter Estimates}

In this section we report the posterior estimates of a few parameters of interest to highlight the properties of the proposed model in (4)-(8). One main question we wish to address is: Is the realized volatility measure a useful estimate of the underlying inflation expectations uncertainty? To answer that question, we plot the prior and posterior densities of $a_{0}$ and $a_{1}$ in Figure 5. Recall that $a_{0}$ and $a_{1}$ are respectively the intercept and slope of the log realized volatility equation in (8). If the log realized volatility is an unbiased measure of the underlying inflation expectations uncertainty, we expect $a_{0}=0$ and $a_{1}=1$.
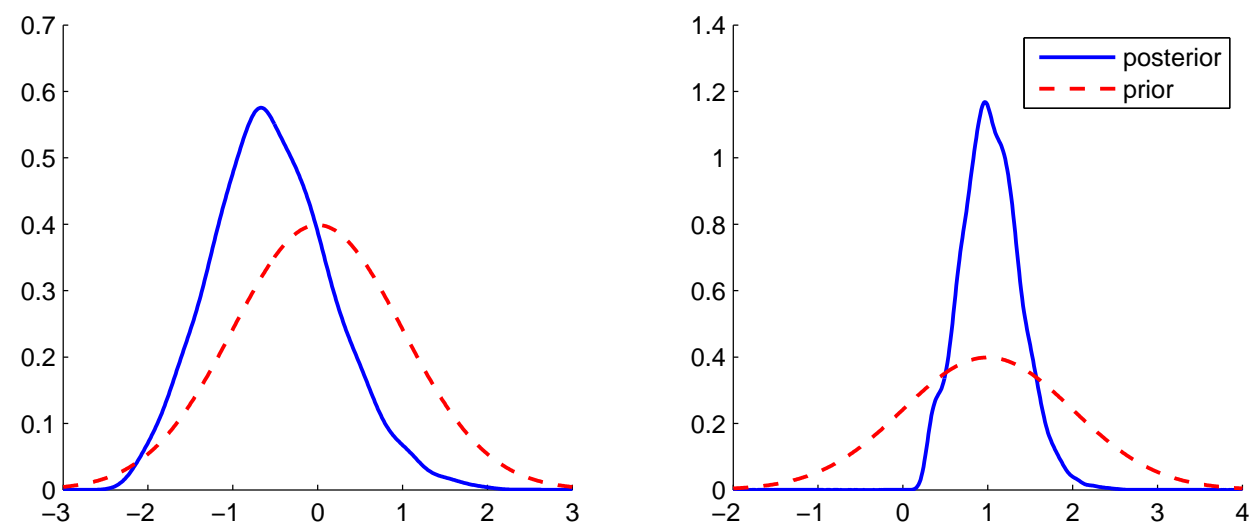

Figure 5: Prior and posterior densities of $a_{0}$ (left panel) and $a_{1}$ (right panel).

The left panel of Figure 5 shows that the posterior density of $a_{0}$ is centered around -0.5 , suggesting some evidence of bias. However, the parameter uncertainty is sufficiently large that the density has substantial mass around 0. In fact, the Bayes factor in favor of the 
hypothesis that $a_{0}=0$ is 0.98 , showing equal evidence in favor and against $a_{0}=0$.

In contrast, the posterior density of $a_{1}$ is centered around 1 and has more mass around that value compared to the prior. The Bayes factor in favor of the hypothesis that $a_{1}=1$ is 2.9 , indicating some evidence that $a_{1}=1$. Overall, we conclude that the realized volatility provides useful information for estimating the inflation expectations uncertainty, but it may not be a perfectly unbiased measure.

Next, we report in Table 1 the posterior estimates of $\sigma_{z}^{2}$, the error variance in the log realized volatility equation. The posterior mean of $\sigma_{z}^{2}$ is 0.66 , compared to the prior mean of 0.3. This variance estimate is relatively large, suggesting that the realized volatility is a noisy measure of the underlying inflation expectations uncertainly. This is also apparent in Figure 1.

Table 1: Posterior estimates of selected parameters.

\begin{tabular}{lccc}
\hline \hline parameter & $a_{0}$ & $a_{1}$ & $\sigma_{z}^{2}$ \\
\hline mean & -0.53 & 1.04 & 0.66 \\
std. dev. & $(0.71)$ & $(0.35)$ & $(0.09)$ \\
\hline \hline
\end{tabular}

\subsection{Model Comparison}

In this section we provide some evidence that the proposed model is favored by the data. Specifically, we compute the marginal likelihood for UCSV, UCSV-RV and UCSVRV-BE. Each marginal likelihood estimate is computed by decomposing the marginal density of the inflation data as the product of predictive likelihoods. In particular, let $\boldsymbol{\pi}_{1: t}=\left(\pi_{1}, \ldots, \pi_{t}\right)^{\prime}$ denote the inflation data up to time $t$. Then, we can factor the marginal likelihood for model $M_{k}$ as follows:

$$
p\left(\boldsymbol{\pi} \mid \mathbf{W}_{k}, M_{k}\right)=p\left(\pi_{1} \mid \mathbf{W}_{1, k}, M_{k}\right) \prod_{t=1}^{T-1} p\left(\pi_{t+1} \mid \boldsymbol{\pi}_{1: t}, \mathbf{W}_{1: t, k}, M_{k}\right)
$$

where $p\left(\pi_{t+1} \mid \boldsymbol{\pi}_{1: t}, \mathbf{W}_{1: t, k}, M_{k}\right)$ is the predictive likelihood and $\mathbf{W}_{1: t, k}$ is the set of additional data up to time $t$ used in model $M_{k}$ (e.g., the realized volatility or the level of breakeven inflation).

Therefore, even though some models use more than inflation data, the marginal likelihoods thus computed are comparable across models. The results are reported in Table 2. 
Table 2: Log marginal likelihood estimates of selected models.

\begin{tabular}{lccc}
\hline \hline & UCSV & UCSV-RV & UCSV-RV-BE \\
\hline $\log$ marginal likelihood & -415 & -412 & -423 \\
\hline \hline
\end{tabular}

Our baseline model UCSV-RV is the best among the three models, showing that adding the realized volatility measure improves the model fit relative to the increase in model complexity. For example, the Bayes factor in favor of UCSV-RV against UCSV is about $20\left(\approx \mathrm{e}^{3}\right)$. In other words, if we assume both models are equally probable a priori, the former is 20 times more likely than the latter given the data. Interestingly, even though the UCSV-RV-BE model has the most information, its performance is worse than even UCSV. This result suggests that there are large discrepancies between the breakeven inflation and the model-based trend inflation.

\subsection{Forecasting Results}

In this section we conduct a recursive forecasting exercise to evaluate the forecast performance of the proposed model in terms of its density forecasts of CPI inflation. To evaluate the $m$-step-ahead density forecast $p\left(\pi_{t+m} \mid \boldsymbol{\pi}_{1: t}, \mathbf{z}_{1: t}\right)$, where $\boldsymbol{\pi}_{1: t}$ and $\mathbf{z}_{1: t}$ are respectively the inflation and realized volatility data up to time $t$, one natural measure is the predictive likelihood $p\left(\pi_{t+m}=\pi_{t+m}^{o} \mid \boldsymbol{\pi}_{1: t}, \mathbf{z}_{1: t}\right)$-i.e., the predictive density of $\pi_{t+m}$ evaluated at the observed value $\pi_{t+m}^{o}$. It is clear that if the actual outcome $\pi_{t+m}^{o}$ is likely under the density forecast, the value of the predictive likelihood will be large, and vise versa. We then summarize the performance of the density forecasts using the sum of log predictive likelihoods:

$$
\sum_{t=t_{0}}^{T-m} \log p\left(\pi_{t+m}=\pi_{t+m}^{\mathrm{o}} \mid \boldsymbol{\pi}_{1: t}, \mathbf{z}_{1: t}\right),
$$

where $t_{0}$ is the time index for the start of the evaluation period. The evaluation period for our forecasting exercise is from January 2005 to December 2015.

For easy comparison, we report in Table 3 the differences of the sum of log predictive likelihoods from those of the UCSV model. Positive values indicate better forecast performance than the benchmark. For all the forecast horizons considered, the proposed UCSV-RV outperforms the UCSV model, whereas UCSV-RV-BE does worse than UCSV in 1- and 4-step-ahead forecasts. 
Table 3: Sums of log predictive likelihoods relative to UCSV.

\begin{tabular}{lcccc}
\hline \hline & 1-step-ahead & 2-step-ahead & 3-step-ahead & 4-step-ahead \\
\hline UCSV-RV & 3.4 & 8.5 & 7.4 & 6.0 \\
UCSV-RV-BE & -7.2 & 0.4 & 1.6 & -1.7 \\
\hline \hline
\end{tabular}

To investigate the forecast performance of UCSV-RV in more detail, we plot in Figure 6 the cumulative sums of log predictive likelihoods over the whole evaluation period (relative to UCSV) for 1- and 4-step-ahead forecasts. It is clear from the figure that UCSV-RV consistently outperforms the benchmark. By contrast, UCSV-RV-BE performs better than UCSV only early in the sample; its performance has deteriorated against UCSV since the onset of the Great Recession. These results again suggest that while one should not interpret the breakeven inflation as purely inflation expectation, the realized volatility constructed from the breakeven inflation is useful in refining estimates of trend inflation volatility, which in turn helps provide better inflation density forecasts.
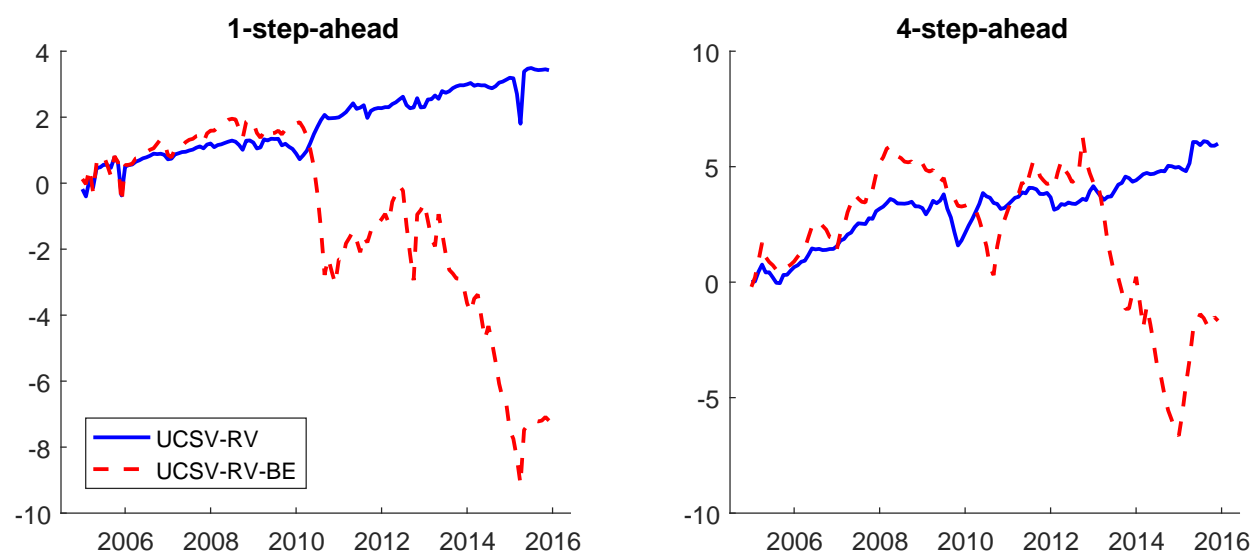

Figure 6: Cumulative sums of log predictive likelihoods relative to UCSV.

\section{Additional Models and Alternative Data Sources}

In this section we present additional results using variations of the proposed model and different data sources. The primary objective of this exercise is to check the robustness of the main results and to investigate the information content of alternative data sources. 


\subsection{Different Model Specifications}

First, we investigate the modeling assumption that the constructed realized volatility does not react to movements in $h_{t}$, the log volatility of the transitory component of inflation. To that end, we consider a version of the UCSV-RV model where (8) is extended to include $h_{t}$ as a covariate:

$$
\log z_{t}=a_{0}+a_{1} g_{t}+a_{2} h_{t}+u_{t}^{z}, \quad u_{t}^{z} \sim \mathcal{N}\left(0, \sigma_{z}^{2}\right)
$$

We call this variant UCSV-RV- $h$. Clearly, this model includes UCSV-RV as a special case with $a_{2}=0$.

Figure 7 plots the posterior density of $a_{2}$. It is clear that the posterior density has substantial mass around small values. In fact, the posterior mean of $a_{2}$ is estimated to be 0.2 with a $90 \%$ credible interval $(-0.11,0.48)$ — which includes 0 . Moreover, the Bayes factor in favor of the hypothesis that $a_{2}=0$ is 1.02 , showing equal evidence in favor and against the hypothesis that $a_{2}=0$.

We plot the estimates of inflation expectations uncertainty from UCSV-RV- $h$ in Figure 7. These estimates are fairly similar to those of our baseline model UCSV-RV. In particular, the inflation expectations uncertainty sharply increases at the onset of the Great Recession, even though the magnitude of the increase is smaller compared to the baseline model.
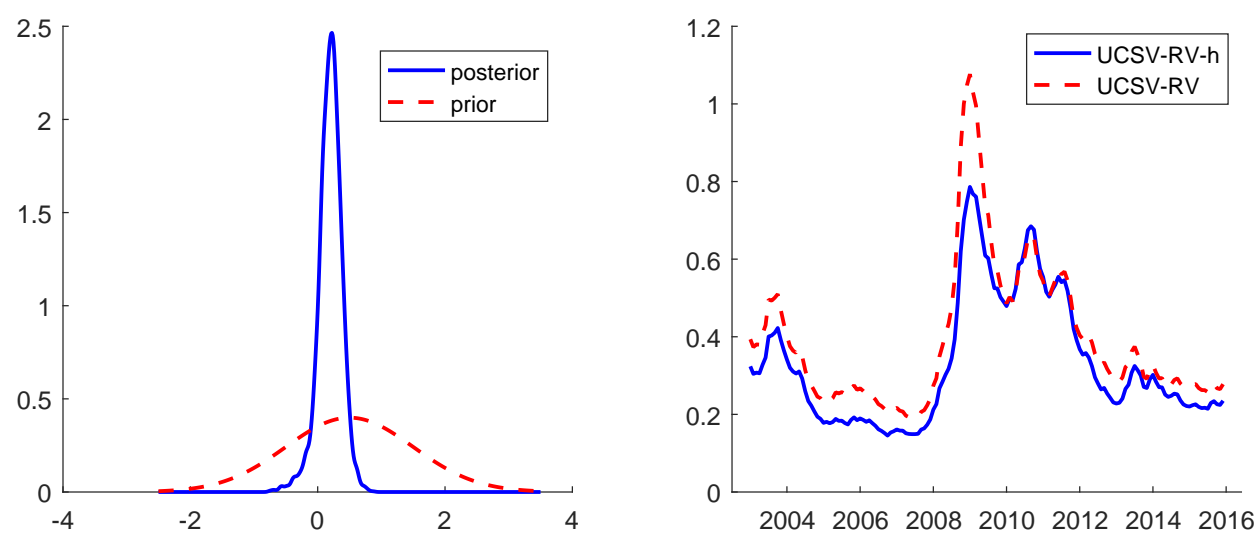

Figure 7: Prior and posterior densities of $a_{2}$ (left panel) and stochastic volatility estimates expressed in standard deviations $\exp \left(g_{t} / 2\right)$ from UCSV-RV and UCSV-RV- $h$ (right panel).

Next, we consider a variant of the baseline model in which the realized volatility equation 
also has stochastic volatility. This is motivated by the concern that the volatility of the realized volatility might be time-varying over the sample period. Specifically, we replace (8) with

$$
\log z_{t}=a_{0}+a_{1} g_{t}+u_{t}^{z}, \quad u_{t}^{z} \sim \mathcal{N}\left(0, \mathrm{e}^{v_{t}}\right),
$$

where the $\log$ volatility $v_{t}$ follows a random walk. This variant is denoted as UCSV-RVSV.

This version has similar support as the baseline UCSV-RV - its log marginal likelihood is also -412. The left panel in Figure 8 reports the posterior densities of $a_{0}$ and $a_{1}$ under UCSV-RV-SV. These estimates are very similar to those of the baseline. In particular, the posterior densities of $a_{0}$ and $a_{1}$ are centered around -0.5 and 1 , respectively. These results confirm the conclusion that while the realized volatility provides useful information for estimating the inflation expectations uncertainty, it may not be an unbiased measure.

Figure 8 also plots the inflation expectations uncertainty under UCSV-RV-SV. The estimates are again broadly similar to those of the baseline, showing that allowing for stochastic volatility in the realized volatility equation does not change our conclusion that the inflation expectations uncertainty exhibits a sharp increase at the onset of the Great Recession.
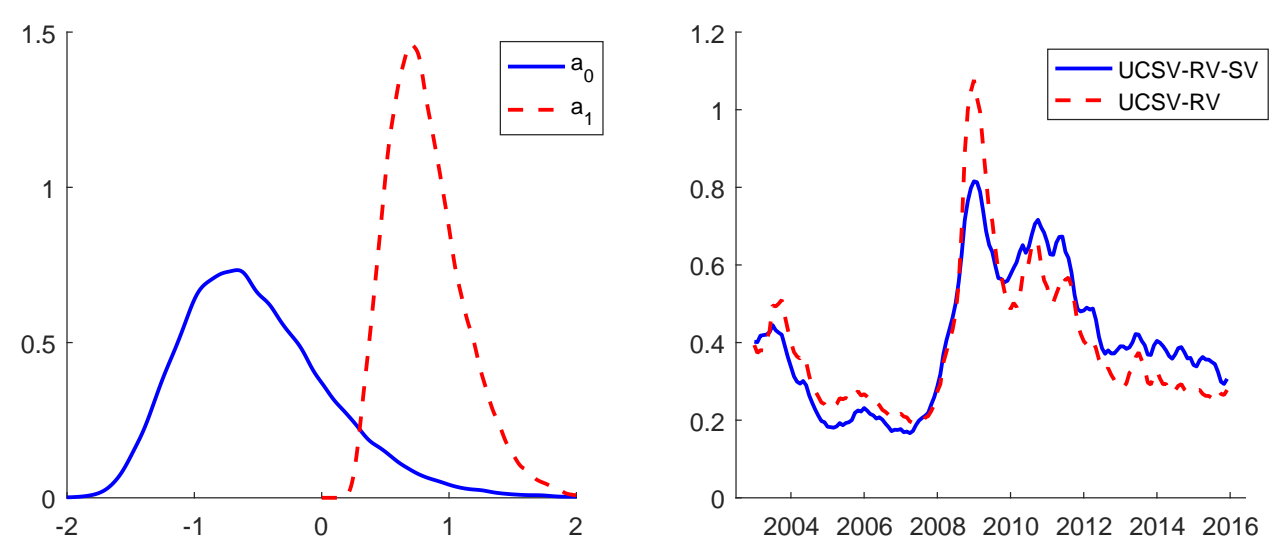

Figure 8: Posterior densities of $a_{0}$ and $a_{1}$ (left panel) and stochastic volatility estimates expressed in standard deviations $\exp \left(g_{t} / 2\right)$ from UCSV-RV and UCSV-RV-SV (right panel).

In the third variant of the baseline, we extend the realized volatility equation (8) to allow for an MA(1) process:

$$
\log z_{t}=a_{0}+a_{1} g_{t}+u_{t}^{z}+\psi u_{t-1}^{z}, \quad u_{t}^{z} \sim \mathcal{N}\left(0, \sigma_{z}^{2}\right),
$$


and we refer to this model as UCSV-RV-MA. This version allows more persistence in the realized volatility. Obviously, if $\psi=0$, then it reduces to UCSV-RV. The posterior density of $\psi$, as shown in the left panel in Figure 9, is centered around 0.2, although there is substantial mass around 0. Consistent with the estimation result, the log marginal likelihood of UCSV-RV-MA is -411 (compared to -412 of the baseline), which shows that this variant is slightly favored by the data. However, the inflation expectations uncertainty estimates under this variant are essentially the same as those of the baseline.
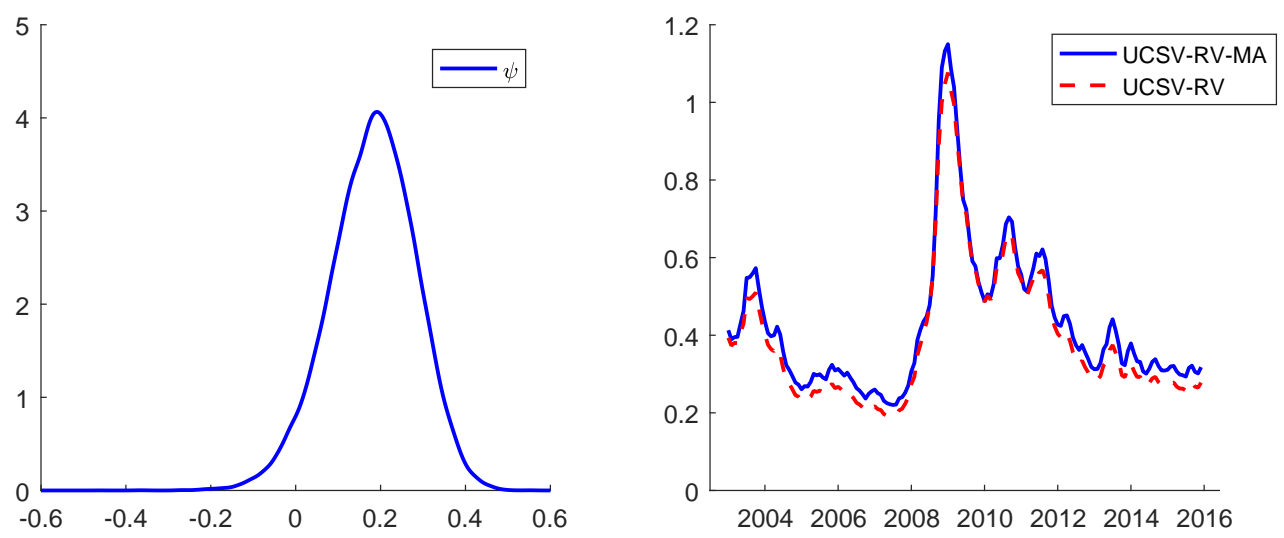

Figure 9: Posterior density of the MA(1) coefficient $\psi$ (left panel) and stochastic volatility estimates expressed in standard deviations $\exp \left(g_{t} / 2\right)$ from UCSV-RV and UCSV-RV-MA (right panel).

\subsection{Accounting for Liquidity Premium}

As mentioned earlier, the breakeven inflation includes not only inflation expectations, but also liquidity risk premium. Given the differential liquidity in the Treasury and TIPS markets at the onset of the Great Recession, one might be concerned that the sharp rise in the constructed realized volatility measure reflects more about changes in liquidity risk premium rather than long-term inflation expectations.

To adjust for the liquidity risk, we follow the approach in Gürkaynak, Sack, and Wright (2010b) to regress the breakeven inflation on a constant and proxies for liquidity. The residuals from this regression are used as raw data to compute an alternative realized volatility series. Specifically, we use the VIX options-implied volatility index, which reflects short-term uncertainty in the Standard \& Poors 500 index, as a proxy. ${ }^{4}$

\footnotetext{
${ }^{4}$ Andreasen, Christensen, and Riddell (2017) identify a few variables that are highly correlated with the TIPS liquidity premium they constructed. In particular, they find that the correlation between the
} 
The regression coefficient corresponding to the VIX index is estimated to be -0.015 with a $95 \%$ confidence interval $(-0.016,-0.014)$. The estimate has the expected sign: high uncertainty tends to increase the risk associated with the future resale price of any security, which increases the required liquidity premium and lowers the breakeven inflation. The $R^{2}$ of the regression is about 0.2 , i.e., if we use VIX as a proxy for liquidity premium, about $20 \%$ of the variation in the breakeven inflation can be attributed to changes in liquidity risk.

This alternative measure of realized volatility is plotted in Figure 10. After adjusting for liquidity premium, there remains an uptick in the realized volatility at the start of 2008 . Next, we rerun the baseline model using this new measure, and the corresponding inflation expectations uncertainty are reported in Figure 10 (UCSV-RV-liq). As the figure shows, the new estimates are essentially the same as the baseline, showing that the variation in liquidity premium does not dominate the constructed realized volatility.
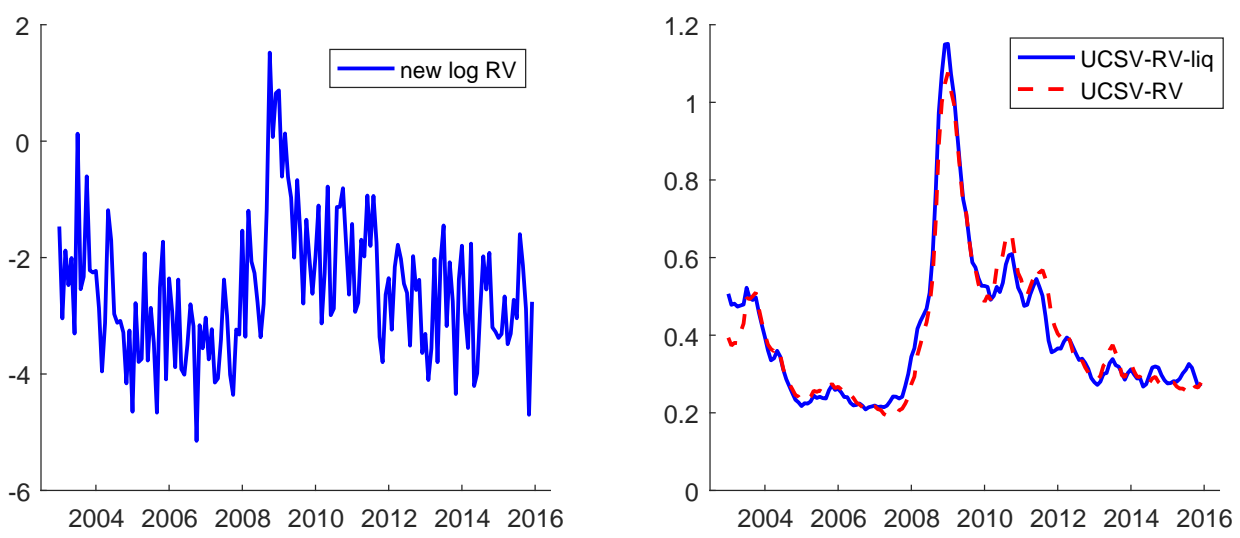

Figure 10: The log realized volatility corrected for liquidity premium (left panel) and stochastic volatility estimates expressed in standard deviations $\exp \left(g_{t} / 2\right)$ from UCSVRV and UCSV-RV-liq (right panel).

\subsection{Inflation Forecast Disagreement}

Next, we investigate the information content of cross-sectional dispersion of survey forecasts as an alternative source of inflation expectations uncertainty. While the forecast disagreement is conceptually distinct from inflation uncertainty, a few papers have investigated their relationships with mixed results (see, e.g., Boero, Smith, and Wallis, 2008; Rich and Tracy, 2010). Here we construct the forecast disagreement variable based on the

VIX index and their liquidity premium is high and has the expected sign (0.67). 
cross-sectional forecast dispersion from the Survey of Professional Forecasters, sourced from the Federal Reserve Bank of Philadelphia. Specifically, the disagreement measure is the interquartile range - difference between the 75 th percentile and the 25 th percentileof the one-year-ahead CPI forecasts. ${ }^{5}$ The data frequency is quarterly and we use the same quarterly value for the three months in the quarter. The disagreement data are plotted in the left panel in Figure 11.

We re-estimate the model (4)-(8) using this disagreement measure instead of the realized volatility $z_{t}$. This variant is denoted as UCSV-D. Recall that for a normal random variable with variance $\sigma^{2}$, its interquartile range is about $1.35 \sigma$. Hence, if this dispersion measure is an unbiased measure of $g_{t}$, the log volatility of the trend inflation, one would expect that $a_{0}=\log 1.35 \approx 0.3$ and $a_{1}=0.5$. On the other hand, if this dispersion measure has no information about $g_{t}$, then $a_{1}$ should be 0 . The posterior means of $a_{0}$ and $a_{1}$ are, respectively, 0.28 and 0.38 with $90 \%$ credible intervals $(-0.16,0.80)$ and $(0.22,0.57)$. The estimated $a_{1}$ is less than 0.5 , but the $90 \%$ credible interval excludes 0 .
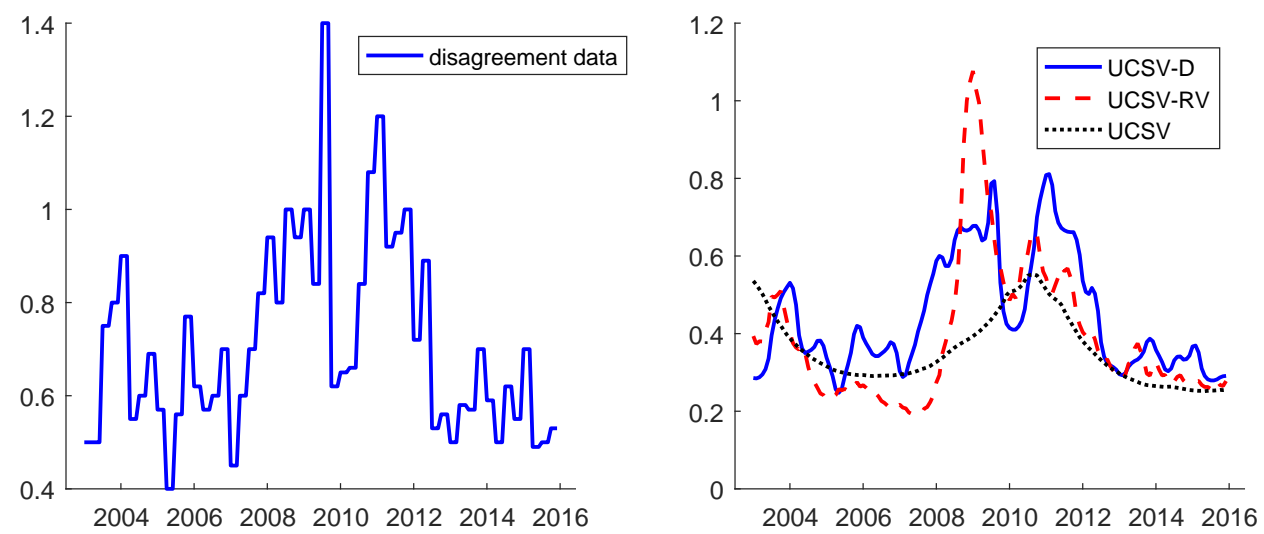

Figure 11: Forecast disagreement (left panel) and stochastic volatility estimates expressed in standard deviations $\exp \left(g_{t} / 2\right)$ from UCSV-RV-D (right panel).

To compare the information content of this disagreement variable with that of the realized volatility measure, we compute the log marginal likelihood of UCSV-D. The estimate is -414, which is slightly less than the corresponding value of -412 under UCSV-RV. These results show that although this disagreement variable contains some information about $g_{t}$, its quality is not as good as the proposed realized volatility measure.

Figure 11 also reports the inflation expectations uncertainty estimates under UCSV-D.

\footnotetext{
${ }^{5}$ Ideally the forecast horizon should be substantially longer to match the uncertainty of long-run inflation expectations, but one-year-ahead is the longest horizon available.
} 
Despite the very different data source, the estimates under UCSV-D are broadly similar to those of the baseline UCSV-RV. In particular, the inflation expectations uncertainty under UCSV-D sharply increases in early 2008. By contrast, under UCSV the inflation expectations uncertainty moves slowly, and peaks only in 2011.

\section{Concluding Remarks and Future Research}

We use daily breakeven inflation to construct a realized measure of inflation expectations volatility. We then incorporate this realized volatility measure in a standard UCSV model to investigate how well it matches the model-based measure of trend inflation volatility. We find that the two measure are largely compatible, and the realized volatility helps refine the estimates of inflation expectations uncertainty and improve inflation forecasts. In addition, we find significant changes in inflation expectations uncertainty during the Great Recession.

In future work, it would be fruitful to have a term structure model to combine multiple realized volatility measures from different horizons. It would also be interesting in future work to study the impact of inflation expectations uncertainty on other macroeconomic variables. 


\section{A Appendix: Estimation Details}

In this appendix we provide the estimation details of fitting the UCSV-RV-BE model defined in (4)-(9) using MCMC methods. The UCSV-RV model is a restricted version where equation (9) is omitted. We implement a Gibbs sampler that sequentially draws from the full conditional distributions of the parameters and the latent states. The parameters are $\sigma_{h}^{2}, \sigma_{g}^{2}, \sigma_{z}^{2}, \sigma_{x}^{2}, \mathbf{a}=\left(a_{0}, a_{1}\right)^{\prime}$ and $\mathbf{b}=\left(b_{0}, b_{1}\right)^{\prime}$, and the latent states are $\mathbf{g}$, $\mathbf{h}$ and $\boldsymbol{\pi}^{*}$.

Let $\mathcal{I} \mathcal{G}\left(c_{1}, c_{2}\right)$ denote the inverse-gamma distribution with mean $c_{1} /\left(c_{2}-1\right)$. We consider the following priors: $\sigma_{h}^{2} \sim \mathcal{I} \mathcal{G}\left(s_{h} / 2, v_{h} / 2\right), \sigma_{g}^{2} \sim \mathcal{I} \mathcal{G}\left(s_{g} / 2, v_{g} / 2\right), \sigma_{z}^{2} \sim \mathcal{I} \mathcal{G}\left(s_{z} / 2, v_{z} / 2\right)$, $\sigma_{x}^{2} \sim \mathcal{I} \mathcal{G}\left(s_{x} / 2, v_{x} / 2\right), \mathbf{a} \sim \mathcal{N}\left(\mathbf{m}_{a}, \mathbf{V}_{a}\right)$ and $\mathbf{b} \sim \mathcal{N}\left(\mathbf{m}_{b}, \mathbf{V}_{b}\right)$. Finally, we initialize the state equations using $g_{1} \sim \mathcal{N}\left(m_{g}, V_{g}\right), h_{1} \sim \mathcal{N}\left(m_{h}, V_{h}\right)$ and $\left(\pi_{1}^{*} \mid g_{1}\right) \sim \mathcal{N}\left(m_{\pi^{*}}, \mathrm{e}^{g_{1}} V_{\pi^{*}}\right)$.

Posterior draws can be obtained by sequentially performing the following MCMC steps:

1. Sample $\mathbf{g}=\left(g_{1}, \ldots, g_{T}\right)^{\prime}$.

To sample the log volatilities, we adopt the auxiliary mixture sampler of Kim, Shepherd, and Chib (1998) by approximating the nonlinear state space model using a mixture of conditionally linear Gaussian state space models with mixture indicators $\mathbf{s}^{g}=\left(s_{1}^{g}, \ldots, s_{T}^{g}\right)^{\prime}$. We first sample the mixture indicators $\mathbf{s}^{g}$ given the current $g$. Then, we draw $g$ given the mixture indicators $\mathbf{s}^{g}$. For a textbook treatment of the auxiliary mixture sampler, see Chan and Hsiao (2014).

First define $y_{1}^{g}=\log \left(\left(\pi_{1}^{*}-m_{\pi^{*}}\right)^{2} / V_{\pi^{*}}\right)$ and $y_{t}^{g}=\log \left(\left(\pi_{t}^{*}-\pi_{t-1}^{*}\right)^{2}\right)$ for $t=2, \ldots, T$. Since $s_{1}^{g}, \ldots, s_{T}^{g}$ are conditionally independent, we can sample them sequentially. Each $s_{t}^{g}$ takes values in $\{1, \ldots, 7\}$ with probabilities

$$
p\left(s_{t}^{g}=k \mid y_{t}^{g}, g_{t}\right) \propto w_{k} f_{N}\left(y_{t}^{g}-g_{t} ; m_{k}, v_{k}^{2}\right) \quad \text { for } k=1,2, \ldots, 7
$$

where $f_{N}\left(\cdot ; u, v^{2}\right)$ is the Gaussian density with mean $u$ and variance $v^{2}$, and the values $\left(w_{k}, m_{k}, v_{k}^{2}\right)$ are given in Table 4 of Kim, Shepherd, and Chib (1998).

Next, we sample $\mathbf{g}$ given the mixture indicators $\mathbf{s}^{g}$. To that end, stack $\mathbf{y}^{g}=\left(y_{1}^{g}, \ldots, y_{T}^{g}\right)^{\prime}$ and rewrite (5) as

$$
\mathbf{y}^{g}=\mathbf{g}+\boldsymbol{\varepsilon}^{g}, \quad \boldsymbol{\varepsilon}^{g} \sim \mathcal{N}\left(\mathbf{d}_{\mathbf{s}^{g}}, \mathbf{\Omega}_{\mathbf{s}^{g}}\right)
$$


with density function

$$
p\left(\mathbf{y}^{g} \mid \mathbf{g}, \mathbf{s}^{g}\right) \propto \exp \left(-\frac{1}{2}\left(\mathbf{y}^{g}-\mathbf{d}_{\mathbf{s}^{g}}-\mathbf{g}\right)^{\prime} \mathbf{\Omega}_{\mathbf{s}^{g}}^{-1}\left(\mathbf{y}^{g}-\mathbf{d}_{\mathbf{s}^{g}}-\mathbf{g}\right)\right),
$$

where $\mathbf{d}_{\mathbf{s}^{g}}$ and $\boldsymbol{\Omega}_{\mathbf{s}^{g}}$ are constant matrices determined by $\mathbf{s}^{g}$, and $\boldsymbol{\Omega}_{\mathbf{s}^{g}}$ is diagonal.

Next, write the state equation (7) in matrix form

$$
\mathbf{H g}=\mathbf{u}^{g}, \quad \mathbf{u}^{g} \sim \mathcal{N}\left(\widetilde{\mathbf{m}}_{g}, \mathbf{S}_{g}\right)
$$

where $\widetilde{\mathbf{m}}_{g}=\left(m_{g}, 0,0, \ldots, 0\right)^{\prime}, \mathbf{S}_{g}=\operatorname{diag}\left(V_{g}, \sigma_{g}^{2}, \ldots, \sigma_{g}^{2}\right)$ and

$$
\mathbf{H}=\left(\begin{array}{ccccc}
1 & 0 & 0 & \cdots & 0 \\
-1 & 1 & 0 & \cdots & 0 \\
0 & -1 & 1 & \cdots & 0 \\
\vdots & \ddots & \ddots & \ddots & \vdots \\
0 & \cdots & 0 & -1 & 1
\end{array}\right)
$$

Hence, the prior density of $\mathbf{g}$ is given by

$$
\begin{aligned}
p\left(\mathbf{g} \mid \sigma_{g}^{2}\right) & \propto \exp \left(-\frac{1}{2}\left(\mathbf{g}-\mathbf{H}^{-1} \widetilde{\mathbf{m}}_{g}\right)^{\prime} \mathbf{H}^{\prime} \mathbf{S}_{g}^{-1} \mathbf{H}\left(\mathbf{g}-\mathbf{H}^{-1} \widetilde{\mathbf{m}}_{g}\right)\right) \\
& =\exp \left(-\frac{1}{2}\left(\mathbf{g}-\mathbf{1}_{T} \mathbf{m}_{g}\right)^{\prime} \mathbf{H}^{\prime} \mathbf{S}_{g}^{-1} \mathbf{H}\left(\mathbf{g}-\mathbf{1}_{T} \mathbf{m}_{g}\right)\right)
\end{aligned}
$$

where $\mathbf{1}_{T}$ is a $T \times 1$ column of ones. Note that the prior mean of $\mathbf{g}$ is $\mathbf{1}_{T} m_{g}$ since $g_{t}$ is a random walk process.

Now, let $\widetilde{\mathbf{z}}=\left(\log z_{1}, \ldots, \log z_{T}\right)^{\prime}$ and rewrite (8) as

$$
\widetilde{\mathbf{z}}=a_{0} \mathbf{1}_{T}+a_{1} \mathbf{g}+\mathbf{u}^{z}, \quad \mathbf{u}^{z} \sim \mathcal{N}\left(\mathbf{0}, \sigma_{z}^{2} \mathbf{I}_{T}\right)
$$

with density function

$$
p\left(\widetilde{\mathbf{z}} \mid \mathbf{g}, \sigma_{z}^{2}, a_{0}, a_{1}\right) \propto \exp \left(-\frac{1}{2 \sigma_{z}^{2}}\left(\widetilde{\mathbf{z}}-a_{0} \mathbf{1}_{T}-a_{1} \mathbf{g}\right)^{\prime}\left(\widetilde{\mathbf{z}}-a_{0} \mathbf{1}_{T}-a_{1} \mathbf{g}\right)\right) .
$$


Finally, the full conditional density of $\mathbf{g}$ is given by

$$
\begin{aligned}
p\left(\mathbf{g} \mid \mathbf{y}^{g}, \widetilde{\mathbf{z}}, \mathbf{s}^{g}, \sigma_{g}^{2}, \sigma_{z}^{2}, a_{0}, a_{1}\right) \propto p\left(\mathbf{y}^{g} \mid \mathbf{g}, \mathbf{s}^{g}\right) p\left(\mathbf{g} \mid \sigma_{g}^{2}\right) p\left(\widetilde{\mathbf{z}} \mid \mathbf{g}, \sigma_{z}^{2}, a_{0}, a_{1}\right) \\
\propto \exp \left\{-\frac{1}{2}\left[\mathbf{g}^{\prime}\left(\mathbf{\Omega}_{\mathbf{s}^{g}}^{-1}+\mathbf{H}^{\prime} \mathbf{S}_{g}^{-1} \mathbf{H}+\frac{a_{1}^{2}}{\sigma_{z}^{2}} \mathbf{I}_{T}\right) \mathbf{g}\right.\right. \\
\left.\left.\quad-2 \mathbf{g}^{\prime}\left(\boldsymbol{\Omega}_{\mathbf{s}^{g}}^{-1}\left(\mathbf{y}^{g}-\mathbf{d}_{\mathbf{s}^{g}}\right)+\mathbf{H}^{\prime} \mathbf{S}_{g}^{-1} \mathbf{H} \mathbf{1}_{T} m_{g}+\frac{a_{1}}{\sigma_{z}^{2}}\left(\widetilde{\mathbf{z}}-a_{0} \mathbf{1}_{T}\right)\right)\right]\right\},
\end{aligned}
$$

which is the kernel of the $\mathcal{N}\left(\widehat{\mathbf{g}}, \mathbf{K}_{\mathrm{g}}^{-1}\right)$ distribution, where

$$
\begin{aligned}
\mathbf{K}_{\mathbf{g}} & =\mathbf{\Omega}_{\mathbf{s}^{g}}^{-1}+\mathbf{H}^{\prime} \mathbf{S}_{g}^{-1} \mathbf{H}+\frac{a_{1}^{2}}{\sigma_{z}^{2}} \mathbf{I}_{T} \\
\widehat{\mathrm{g}} & =\mathbf{K}_{\mathbf{g}}^{-1}\left(\boldsymbol{\Omega}_{\mathbf{s}^{g}}^{-1}\left(\mathbf{y}^{g}-\mathbf{d}_{\mathbf{s}^{g}}\right)+\mathbf{H}^{\prime} \mathbf{S}_{g}^{-1} \mathbf{H} \mathbf{1}_{T} m_{g}+\frac{a_{1}}{\sigma_{z}^{2}}\left(\widetilde{\mathbf{z}}-a_{0} \mathbf{1}_{T}\right)\right) .
\end{aligned}
$$

Notice that $\mathbf{H}^{\prime} \mathbf{S}_{\mathbf{g}}^{-1} \mathbf{H} \mathbf{1}_{T} m_{g}=\left(V_{g}^{-1} m_{g}, 0,0, \ldots, 0\right)^{\prime}$. Since $\mathbf{K}_{\mathbf{g}}$ is a band precision matrix, the precision sampler in Chan and Jeliazkov (2009) can be used to sample $\mathbf{g}$ efficiently.

2. Sample $\mathbf{h}=\left(h_{1}, \ldots, h_{T}\right)^{\prime}$.

Similar to the previous step, we implement the auxiliary mixture sampler of Kim, Shepherd, and Chib (1998) by first drawing the mixture indicators $\mathbf{s}^{h}=\left(s_{1}^{h}, \ldots, s_{T}^{h}\right)^{\prime}$ given $\mathbf{h}$ and other parameters, followed by sampling $\mathbf{h}$ given the mixture indicators $\mathbf{s}^{h}$. To that end, define $y_{t}^{h}=\log \left(\left(\pi_{t}-\pi_{t}^{*}\right)^{2}\right)$ and stack $\mathbf{y}^{h}=\left(y_{1}^{h}, \ldots, y_{T}^{h}\right)^{\prime}$. Then, each $s_{t}^{h}$ can be drawn from the conditional posterior distribution as before.

Next, rewrite (4) in matrix form:

$$
\mathbf{y}^{h}=\mathbf{h}+\varepsilon^{h}, \quad \varepsilon^{h} \sim \mathcal{N}\left(\mathbf{d}_{\mathbf{s}^{h}}, \Omega_{\mathbf{s}^{h}}\right),
$$

where $\mathbf{d}_{s^{h}}$ and $\Omega_{\mathbf{s}^{h}}$ are constant matrices determined by $\mathbf{s}^{h}$. Similarly, rewrite (6) as

$$
\mathbf{H h}=\mathbf{u}^{h}, \quad \mathbf{u}^{h} \sim \mathcal{N}\left(\widetilde{\mathbf{m}}_{h}, \mathbf{S}_{h}\right)
$$

where $\widetilde{\mathbf{m}}_{h}=\left(m_{h}, 0,0, \ldots, 0\right)^{\prime}$ and $\mathbf{S}_{h}=\operatorname{diag}\left(V_{h}, \sigma_{h}^{2}, \ldots, \sigma_{h}^{2}\right)$. Using a similar derivation as in Step 1, we have

$$
\left(\mathbf{h} \mid \mathbf{y}^{h}, \mathbf{s}^{h}, \sigma_{h}^{2}\right) \sim \mathcal{N}\left(\widehat{\mathbf{h}}, \mathbf{K}_{\mathbf{h}}^{-1}\right),
$$

where $\mathbf{K}_{\mathbf{h}}=\boldsymbol{\Omega}_{\mathbf{s}^{h}}^{-1}+\mathbf{H}^{\prime} \mathbf{S}_{h}^{-1} \mathbf{H}$ and $\widehat{\mathbf{h}}=\mathbf{K}_{h}^{-1}\left(\boldsymbol{\Omega}_{\mathbf{s}^{h}}^{-1}\left(\mathbf{y}^{h}-\mathbf{d}_{\mathbf{s}^{h}}\right)+\mathbf{H}^{\prime} \mathbf{S}_{h}^{-1} \mathbf{H} \mathbf{1}_{T} m_{h}\right)$.

3. Sample $\boldsymbol{\pi}^{*}=\left(\pi_{1}^{*}, \ldots, \pi_{T}^{*}\right)^{\prime}$. 
Information about $\boldsymbol{\pi}^{*}$ comes from three sources: the two measurement equations (4) and (9), as well as the state equation (5). First, rewrite the three equations as

$$
\begin{aligned}
\boldsymbol{\pi} & =\boldsymbol{\pi}^{*}+\mathbf{u}^{\pi}, & \mathbf{u}^{\pi} & \sim \mathcal{N}\left(\mathbf{0}, \mathbf{S}_{\pi}\right), \\
\mathbf{x} & =b_{0} \mathbf{1}_{T}+b_{1} \boldsymbol{\pi}^{*}+\mathbf{u}^{x}, & \mathbf{u}^{x} & \sim \mathcal{N}\left(\mathbf{0}, \sigma_{x}^{2} \mathbf{I}_{T}\right), \\
\mathbf{H} \boldsymbol{\pi}^{*} & =\mathbf{u}^{\pi^{*}}, & \mathbf{u}^{\pi^{*}} & \sim \mathcal{N}\left(\widetilde{\mathbf{m}}_{\pi^{*}}, \mathbf{S}_{\pi^{*}}\right),
\end{aligned}
$$

where $\mathbf{S}_{\pi}=\operatorname{diag}\left(\mathrm{e}^{h_{1}}, \ldots, \mathrm{e}^{h_{T}}\right), \widetilde{\mathbf{m}}_{\pi^{*}}=\left(m_{\pi^{*}}, 0, \ldots, 0\right)^{\prime}$ and $\mathbf{S}_{\pi^{*}}=\operatorname{diag}\left(\mathrm{e}^{g_{1}} V_{\pi^{*}}, \mathrm{e}^{g_{2}}, \ldots, \mathrm{e}^{g_{T}}\right)$. Using a similar derivation as in Step 1, we have

$$
\left(\boldsymbol{\pi}^{*} \mid \boldsymbol{\pi}, \mathbf{g}, \mathbf{h}, \mathbf{x}, \mathbf{b}\right) \sim \mathcal{N}\left(\widehat{\boldsymbol{\pi}}^{*}, \mathbf{K}_{\boldsymbol{\pi}^{*}}^{-1}\right)
$$

where

$$
\mathbf{K}_{\pi^{*}}=\mathbf{H}^{\prime} \mathbf{S}_{\pi^{*}}^{-1} \mathbf{H}+\mathbf{S}_{\pi}^{-1}+\frac{b_{1}^{2}}{\sigma_{x}^{2}} \mathbf{I}_{T}, \quad \widehat{\boldsymbol{\pi}}^{*}=\mathbf{K}_{\boldsymbol{\pi}^{*}}^{-1}\left(\mathbf{H}^{\prime} \mathbf{S}_{\pi^{*}}^{-1} \widetilde{\mathbf{m}}_{\pi^{*}}+\mathbf{S}_{\pi}^{-1} \boldsymbol{\pi}+\frac{b_{1}}{\sigma_{x}^{2}}\left(\mathbf{x}-\mathbf{1}_{T} b_{0}\right)\right) .
$$

Note that $\mathbf{H}^{\prime} \mathbf{S}_{\pi^{*}}^{-1} \widetilde{\mathbf{m}}_{\boldsymbol{\pi}^{*}}=\left(m_{\pi^{*}} /\left(\mathrm{e}^{g_{1}} V_{\pi^{*}}\right), 0, \ldots, 0\right)^{\prime}$. Again $\mathbf{K}_{\boldsymbol{\pi}^{*}}$ is a band precision matrix, we use the algorithm in Chan and Jeliazkov (2009) to sample $\boldsymbol{\pi}^{*}$.

\section{Sample $\sigma_{g}^{2}, \sigma_{h}^{2}, \sigma_{z}^{2}$ and $\sigma_{x}^{2}$.}

This step is standard as $\sigma_{g}^{2}, \sigma_{h}^{2}, \sigma_{z}^{2}$ and $\sigma_{x}^{2}$ are conditionally independent and each follows an inverse-gamma distribution. Define $\Delta \mathbf{g}=\left(g_{2}-g_{1}, g_{3}-g_{2}, \ldots, g_{T}-g_{T-1}\right)^{\prime}, \Delta \mathbf{h}=$ $\left(h_{2}-h_{1}, h_{3}-h_{2}, \ldots, h_{T}-h_{T-1}\right)^{\prime}, \mathbf{u}^{z}=\widetilde{\mathbf{z}}-a_{0} \mathbf{1}_{T}-a_{1} \mathbf{g}$ and $\mathbf{u}^{x}=\mathbf{x}-b_{0} \mathbf{1}_{T}-b_{1} \boldsymbol{\pi}^{*}$. Then, we have

$$
\begin{aligned}
\sigma_{h}^{2} & \sim \mathcal{I} \mathcal{G}\left(\widehat{s}_{h} / 2, \widehat{v}_{h} / 2\right), \\
\sigma_{g}^{2} & \sim \mathcal{I} \mathcal{G}\left(\widehat{s}_{g} / 2, \widehat{v}_{g} / 2\right), \\
\sigma_{z}^{2} & \sim \mathcal{I} \mathcal{G}\left(\widehat{s}_{z} / 2, \widehat{v}_{z} / 2\right), \\
\sigma_{x}^{2} & \sim \mathcal{I} \mathcal{G}\left(\widehat{s}_{x} / 2, \widehat{v}_{x} / 2\right),
\end{aligned}
$$

where

$$
\begin{array}{ll}
\widehat{s}_{h}=s_{h}+(\Delta \mathbf{h})^{\prime} \Delta \mathbf{h} & \widehat{v}_{h}=v_{h}+T-1, \\
\widehat{s}_{g}=s_{g}+(\Delta \mathbf{g})^{\prime} \Delta \mathbf{g} & \widehat{v}_{g}=v_{g}+T-1, \\
\widehat{s}_{z}=s_{z}+\left(\mathbf{u}^{z}\right)^{\prime} \mathbf{u}^{z} & \widehat{v}_{z}=v_{z}+T, \\
\widehat{s}_{x}=s_{x}+\left(\mathbf{u}^{x}\right)^{\prime} \mathbf{u}^{x} & \widehat{v}_{x}=v_{x}+T .
\end{array}
$$


5. Sample $\mathbf{a}=\left(a_{0}, a_{1}\right)^{\prime}$.

This step is standard since $\left(\mathbf{a} \mid z, g, \sigma_{z}^{2}\right) \sim \mathcal{N}\left(\widehat{\mathbf{a}}, \mathbf{K}_{\mathbf{a}}^{-1}\right)$, where

$$
\mathbf{K}_{\mathbf{a}}=\mathbf{V}_{a}^{-1}+\frac{1}{\sigma_{z}^{2}} \mathbf{X}_{a}^{\prime} \mathbf{X}_{a}, \quad \widehat{\mathbf{a}}=\mathbf{K}_{\mathbf{a}}^{-1}\left(\mathbf{V}_{a}^{-1} \mathbf{m}_{a}+\frac{1}{\sigma_{z}^{2}} \mathbf{X}_{a}^{\prime} \widetilde{\mathbf{z}}\right)
$$

with $\widetilde{\mathbf{z}}=\left(\log z_{1}, \ldots, \log z_{T}\right)^{\prime}$ and $\mathbf{X}_{a}=\left[\mathbf{1}_{T}, \mathbf{g}\right]$ is constructed by stacking the regressors.

6. Sample $\mathbf{b}=\left(b_{0}, b_{1}\right)^{\prime}$.

Similarly, let $\mathbf{X}_{b}=\left[\mathbf{1}_{T}, \boldsymbol{\pi}^{*}\right]$. Then, we have $\left(\mathbf{b} \mid \mathbf{x}, \boldsymbol{\pi}^{*}, \sigma_{x}^{2}\right) \sim \mathcal{N}\left(\widehat{\mathbf{b}}, \mathbf{K}_{\mathbf{b}}^{-1}\right)$, where

$$
\mathbf{K}_{\mathbf{b}}=\mathbf{V}_{b}^{-1}+\frac{1}{\sigma_{x}^{2}} \mathbf{X}_{b}^{\prime} \mathbf{X}_{b}, \quad \widehat{\mathbf{1}}=\mathbf{K}_{\mathbf{b}}^{-1}\left(\mathbf{V}_{b}^{-1} \mathbf{m}_{b}+\frac{1}{\sigma_{x}^{2}} \mathbf{X}_{b}^{\prime} \mathbf{x}\right)
$$




\section{B Appendix: Additional Results}

In this appendix we re-estimate the models using another measure of realized volatility. Specifically, the realized volatility is computed from the the breakeven inflation ei ${ }^{(7,10)}$. The results are reported in Figure 12 to Figure 14. The conclusions we draw from these results are the same from the baseline case: the realized volatility provides useful information for the underlying inflation expectations uncertainty and typically helps refine the estimates.
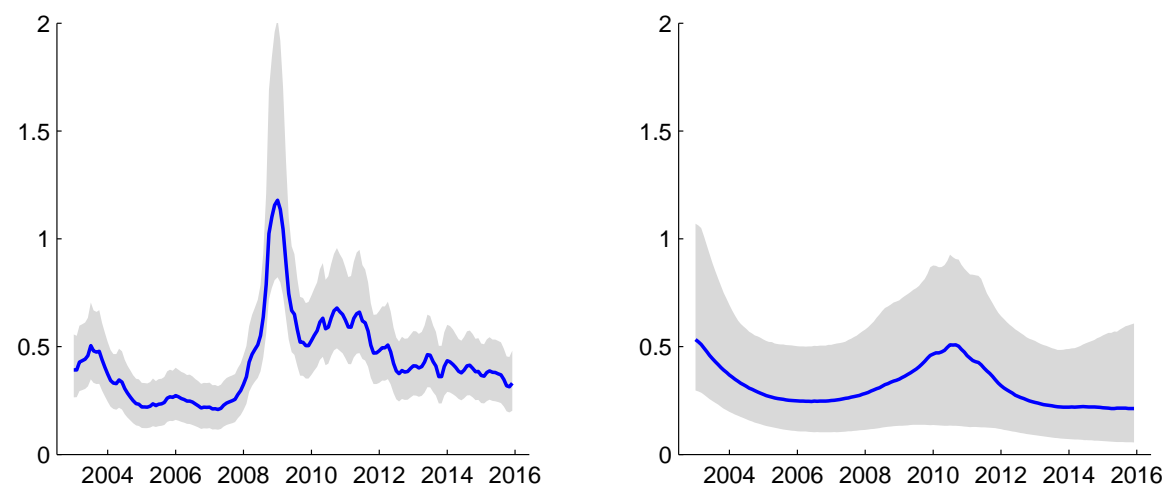

Figure 12: Stochastic volatility estimates expressed in standard deviations $\exp \left(g_{t} / 2\right)$ from UCSV-RV (left panel) and UCSV (right panel). The shaded areas represent the 16- and 84-percentiles. The realized volatility is computed from the the breakeven inflation ei ${ }^{(7,10)}$.

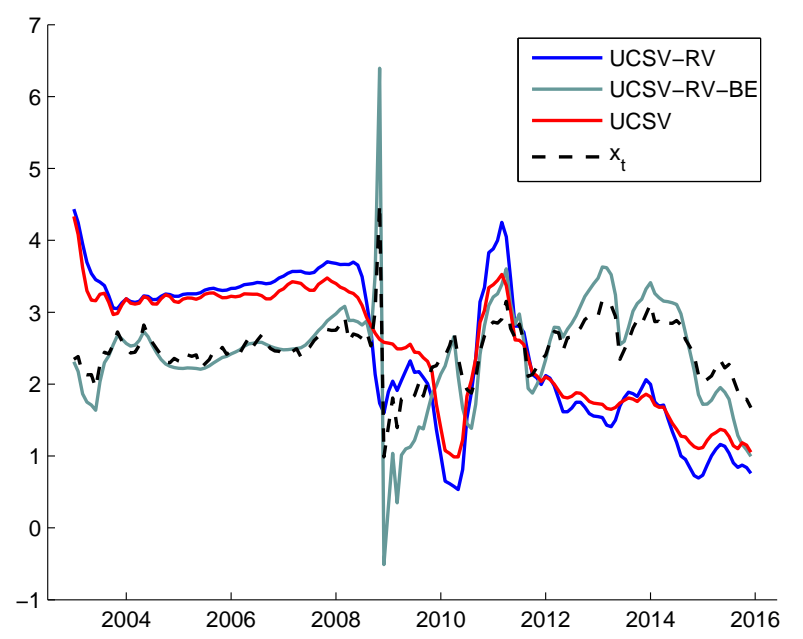

Figure 13: Trend estimates from UCSV-RV, UCSV-RV-BE and UCSV. The realized volatility is computed from the the breakeven inflation $\mathrm{ei}^{(7,10)}$. 

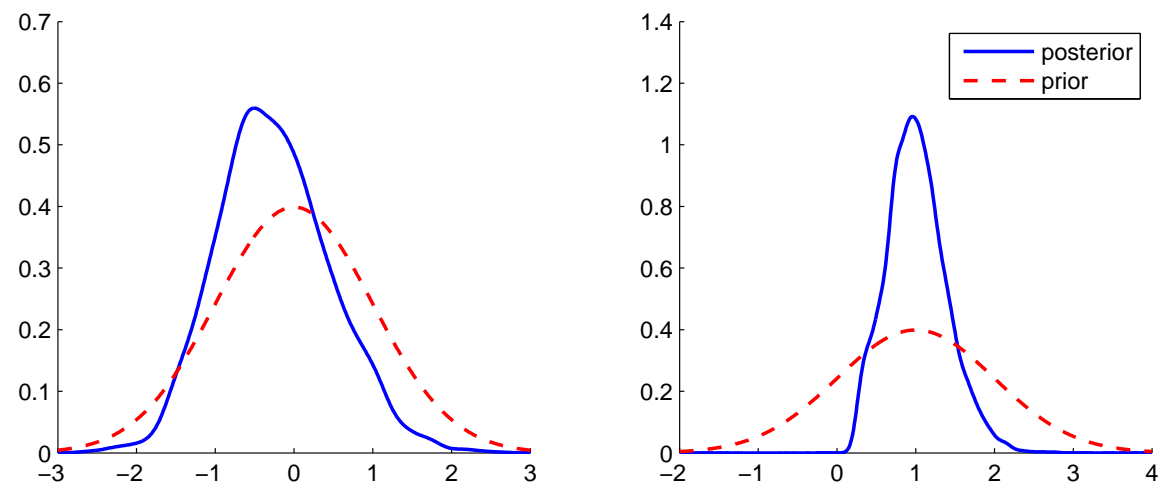

Figure 14: Prior and posterior densities of $a_{0}$ (left panel) and $a_{1}$ (right panel). The realized volatility is computed from the the breakeven inflation ei ${ }^{(7,10)}$. 


\section{References}

Torben G. Andersen, Tim Bollerslev, Francis X. Diebold, and Paul Labys. Modeling and forecasting realized volatility. Econometrica, 71(2):579-625, 2003.

Martin M. Andreasen, Jens H. E. Christensen, and Simon Riddell. The TIPS liquidity premium. Federal Reserve Bank of San Francisco Working Paper Series, 2017.

Ole E. Barndorff-Nielsen and Neil Shephard. Estimating quadratic variation using realized variance. Journal of Applied Econometrics, 17(5):457-477, 2002a.

Ole E. Barndorff-Nielsen and Neil Shephard. Estimating quadratic variation using realized variance. Journal of Applied Econometrics, 17(5):457-477, 2002b.

Ole E. Barndorff-Nielsen and Neil Shephard. Power and bipower variation with stochastic volatility and jumps. Journal of financial econometrics, 2(1):1-37, 2004.

Ole E. Barndorff-Nielsen, Peter R. Hansen, Asger Lunde, and Neil Shephard. Designing realized kernels to measure the ex post variation of equity prices in the presence of noise. Econometrica, 76(6):1481-1536, 2008.

Ole E. Barndorff-Nielsen, Peter R. Hansen, Asger Lunde, and Neil Shephard. Multivariate realised kernels: consistent positive semi-definite estimators of the covariation of equity prices with noise and non-synchronous trading. Journal of Econometrics, 162(2):149169, 2011.

Hakan Berument, Yeliz Yalcin, and Julide Yildirim. The effect of inflation uncertainty on inflation: Stochastic volatility in mean model within a dynamic framework. Economic Modelling, 26(6):1201-1207, 2009.

Stephen Beveridge and Charles R. Nelson. A new approach to decomposition of economic time series into permanent and transitory components with particular attention to measurement of the 'business cycle'. Journal of Monetary Economics, 7(2):151 - 174, 1981.

Nicholas Bloom. The impact of uncertainty shocks. Econometrica, 77(3):623-685, 2009.

Nicholas Bloom. Fluctuations in uncertainty. Technical report, National Bureau of Economic Research, 2013. 
Gianna Boero, Jeremy Smith, and Kenneth F. Wallis. Uncertainty and disagreement in economic prediction: the Bank of England Survey of External Forecasters. The Economic Journal, 118(530):1107-1127, 2008.

Giovanni Caggiano, Efrem Castelnuovo, and Nicolas Groshenny. Uncertainty shocks and unemployment dynamics in US recessions. Journal of Monetary Economics, 67:78-92, 2014.

Joshua C. C. Chan. The stochastic volatility in mean model with time-varying parameters: An application to inflation modeling. Journal of Business and Economic Statistics, 35(1):17-28, 2017.

Joshua C. C. Chan and Cody Y. L. Hsiao. Estimation of stochastic volatility models with heavy tails and serial dependence. In I. Jeliazkov and X.-S. Yang, editors, Bayesian Inference in the Social Sciences. John Wiley \& Sons, Hoboken, 2014.

Joshua C. C. Chan and Ivan Jeliazkov. Efficient simulation and integrated likelihood estimation in state space models. International Journal of Mathematical Modelling and Numerical Optimisation, 1:101-120, 2009.

Joshua C. C. Chan, Gary Koop, and Simon M. Potter. A new model of trend inflation. Journal of Business and Economic Statistics, 31(1):94-106, 2013.

Joshua C. C. Chan, Todd E. Clark, and Gary Koop. A new model of inflation, trend inflation, and long-run inflation expectations. Journal of Money, Credit and Banking, 2017. Forthcoming.

Jens Christensen, Jose Lopez, and Glenn Rudebusch. Inflation expectations and risk premiums in an arbitrage-free model of nominal and real bond yields. Journal of Money, Credit and Banking, 42(s1):143-178, 2010.

Todd E. Clark and Taeyoung Doh. Evaluating alternative models of trend inflation. International Journal of Forecasting, 30(3):426-448, 2014.

Jon Faust and Jonathan Wright. Forecasting inflation. In Graham Elliott and Andrew Timmermann, editors, Handbook of Economic Forecasting, volume 2A, pages 3-56. Elsevier, Amsterdam, 2013.

Olesya V. Grishchenko and Jing-Zhi Huang. The inflation risk premium: Evidence from the TIPS market. In Midwest Finance Association 2013 Annual Meeting Paper, 2012. 
Refet S. Gürkaynak, Andrew Levin, and Eric Swanson. Does inflation targeting anchor long-run inflation expectations? Evidence from the U.S., UK, and Sweden. Journal of the European Economic Association, 8(6):1208-1242, 2010a.

Refet S. Gürkaynak, Brian Sack, and Jonathan H. Wright. The TIPS yield curve and inflation compensation. American Economic Journal: Macroeconomics, 2(1):70-92, 2010b.

Markus Jochmann, Gary Koop, and Simon M. Potter. Modeling the dynamics of inflation compensation. Journal of Empirical Finance, 17(1):157 - 167, 2010.

Kyle Jurado, Sydney C. Ludvigson, and Serena Ng. Measuring uncertainty. The American Economic Review, 105(3):1177-1216, 2015.

Sangjoon Kim, Neil Shepherd, and Siddhartha Chib. Stochastic volatility: Likelihood inference and comparison with ARCH models. Review of Economic Studies, 65(3): 361-393, 1998.

Sharon Kozicki and Peter A. Tinsley. Effective use of survey information in estimating the evolution of expected inflation. Journal of Money, Credit and Banking, 44(1):145-169, 2012 .

Elmar Mertens. Measuring the level and uncertainty of trend inflation. Review of Economics and Statistics, 98(5):950-967, 2016.

Haroon Mumtaz and Konstantinos Theodoridis. The international transmission of volatility shocks: An empirical analysis. Journal of the European Economic Association, 13 (3):512-533, 2015.

James Nason and Gregor Smith. Measuring the slowly evolving trend in US inflation with professional forecasts. CAMA Working Paper 7/2014, 2013.

Simon Potter and Joshua Rosenberg. Are US inflation expectations anchored, contained, or unmoored? manuscript, 2007.

Robert Rich and Joseph Tracy. The relationships among expected inflation, disagreement, and uncertainty: Evidence from matched point and density forecasts. The Review of Economics and Statistics, 92(1):200-207, 2010.

James H. Stock and Mark W. Watson. Why has U.S. inflation become harder to forecast? Journal of Money Credit and Banking, 39:3-33, 2007. 
Jonathan Wright. Evaluating real time VAR forecasts with an informative democratic prior. Journal of Applied Econometrics, 28:762-776, 2013. 